EGU21-3320

https://doi.org/10.5194/egusphere-egu21-3320

EGU General Assembly 2021

(c) Author(s) 2021. This work is distributed under

the Creative Commons Attribution 4.0 License.

\title{
Localization of actinide-bearing particles in sediment samples from the Fukushima restriction zone
}

\author{
Aurélie Diacre $^{1,2}$, Pascal Fichet ${ }^{3}$, Paul Sardini ${ }^{4}$, Jérôme Donnard ${ }^{5}$, Anne-Laure Fauré ${ }^{1}$, Olivier \\ Marie $^{1}$, Katsumi Shozugawa ${ }^{6}$, Michael Susset ${ }^{1}$, Mayumi Hori ${ }^{6}$, Fabien Pointurier ${ }^{1}$, and Olivier \\ Evrard $^{2}$ \\ ${ }^{1}$ Commissariat à l'Energie Atomique et aux énergies alternatives (CEA, DAM, DIF), F-91297 Arpajon, France \\ (aurelie.diacre@lsce.ipsl.fr) \\ ${ }^{2}$ Laboratoire des Sciences du Climat et de l'Environnement (LSCE/IPSL), Unité Mixte de Recherche 8212 (CEA/ CNRS/UVSQ), \\ Université Paris-Saclay, Gif-sur-Yvette, France \\ ${ }^{3}$ Université Paris-Saclay, CEA, Service d'Études Analytiques et de Réactivité des Surfaces, 91191, Gif-sur-Yvette, France \\ ${ }^{4}$ Université Paris-Saclay, CEA, Service d'Études Analytiques et de Réactivité des Surfaces, 91191, Gif-sur-Yvette, France \\ ${ }^{5} \mathrm{Ai} 4 \mathrm{r}, 2$ Rue Alfred Kastler, 44307 Nantes, France \\ ${ }^{6}$ The University of Tokyo, Graduate School of Arts and Sciences, Tokyo 153-8902, Japan
}

The Fukushima Dai-Ichi Nuclear Power Plant (FDNPP) accident that occurred in March 2011 released significant quantities of radionuclides into the environment. Ten years after the accident, questions still remain, particularly about the processes that led to the partial core meltdown of reactors 1 and 3 . So far, some answers have been provided by the investigation of particles containing caesium (Martin et al., 2020) and sometimes uranium (Ochiai et al., 2018). Indeed, the composition of particles, which were produced and spread at the time of the reactor explosion, reflect the conditions that prevailed in the reactor. Accordingly, the objective of the current research is to develop a method for specifically locating actinide-bearing particles in sediment samples collected in the vicinity of FDNPP. To identify and locate such particles, three already existing methods have been upgraded, including 1) the method of fission tracks already used in the field of non-proliferation studies, 2) the autoradiography through the use of imaging plates that are currently employed in the context of the localization of particles containing radio-caesium and the dismantling of nuclear facilities (Haudebourg and Fichet, 2016), and 3) a real time autoradiography method through the use of the BeaQuant ${ }^{\circledR}$ instrument which has been developed for detecting radioactive particles in biology and geosciences.

In this study, a sediment sample collected nearby FDNPP, which may contain particles containing both radio-caesium and actinides, was selected. This sample was dried and sieved to $63 \mu \mathrm{m}$ before being processed according to the different analysis protocols. A quality control sample containing only uranium oxide particles was also analysed, as these particles are devoid of gamma-emitters.

The first results of this comparison of autoradiography methods for the detection of actinidebearing particles in Fukushima samples will be presented. The method of fission tracks was particularly efficient for detecting both natural and anthropogenic uranium. 
The next steps of this study will be to implement this method identified as optimal to isolate and characterise a larger number of particles released by FDNPP. The full characterization of these particles (size, morphology, elemental and isotopic compositions) will provide novel insights to determine their origin and to improve our understanding of their formation processes within the reactors and anticipate their fate in the environment.

\section{References:}

Haudebourg, R., Fichet, P., 2016. A non-destructive and on-site digital autoradiography-based tool to identify contaminating radionuclide in nuclear wastes and facilities to be dismantled. J. Radioanal. Nucl. Chem. 309, 551-561. https://doi.org/10.1007/s10967-015-4610-7

Martin, P.G., Jones, C.P., Cipiccia, S., Batey, D.J., Hallam, K.R., Satou, Y., Griffiths, I., Rau, C., Richards, D.A., Sueki, K., Ishii, T., Scott, T.B., 2020. Compositional and structural analysis of Fukushimaderived particulates using high-resolution $x$-ray imaging and synchrotron characterisation techniques. Sci. Rep. 10, 1636. https://doi.org/10.1038/s41598-020-58545-y

Ochiai, A., Imoto, J., Suetake, M., Komiya, T., Furuki, G., Ikehara, R., Yamasaki, S., Law, G.T.W., Ohnuki, T., Grambow, B., Ewing, R.C., Utsunomiya, S., 2018. Uranium Dioxides and Debris Fragments Released to the Environment with Cesium-Rich Microparticles from the Fukushima Daiichi Nuclear Power Plant. Environ. Sci. Technol. 52, 2586-2594. https://doi.org/10.1021/acs.est.7b06309 